\title{
Molecular dynamics study of accelerated ion-induced shock waves in biological media
}

\author{
Pablo de Vera ${ }^{1,2,3, a}$, Nigel J. Mason ${ }^{2}$, Fred J. Currell ${ }^{1}$, and Andrey V. Solov'yov ${ }^{3, b}$ \\ 1 School of Mathematics and Physics, Queen's University Belfast, BT7 1NN Belfast, Northern Ireland, UK \\ 2 Department of Physical Sciences, The Open University, Walton Hall, MK7 6AA Milton Keynes, England, UK \\ 3 MBN Research Center, Altenhöferallee 3, 60438 Frankfurt am Main, Germany
}

Received 20 April 2016 / Received in final form 28 June 2016

Published online 6 September 2016

(C) The Author(s) 2016. This article is published with open access at Springerlink.com

\begin{abstract}
We present a molecular dynamics study of the effects of carbon- and iron-ion induced shock waves in DNA duplexes in liquid water. We use the CHARMM force field implemented within the MBN Explorer simulation package to optimize and equilibrate DNA duplexes in liquid water boxes of different sizes and shapes. The translational and vibrational degrees of freedom of water molecules are excited according to the energy deposited by the ions and the subsequent shock waves in liquid water are simulated. The pressure waves generated are studied and compared with an analytical hydrodynamics model which serves as a benchmark for evaluating the suitability of the simulation boxes. The energy deposition in the DNA backbone bonds is also monitored as an estimation of biological damage, something which is not possible with the analytical model.
\end{abstract}

\section{Introduction}

Collisional phenomena between fast ions and biomolecules is a topic of major interest since it is necessary to understand the mechanisms of such processes if we are to further exploit ion beam cancer therapy (IBCT). In this therapy technique energetic protons or heavier ions are used clinically to treat deeply seated tumors [1]. The Bragg peak is a feature of ion's propagation in the medium, consisting on a sharp maximum in the depth-dose curve at the end of the incident ions trajectories (contrary to photon or electron beams which have a quite broad energy deposition profile). This feature is advantageous for IBCT from a macroscopic point of view, since energy deposition in the tumor is maximized while surrounding healthy tissue is largely undamaged. However, it is well-known that the effectiveness of IBCT relies on nanoscopic phenomena rather than on macroscopic characteristics [2,3], the former being directly related to atomic collisions with biomolecules. Indeed, a given dose deposited by ions presents a much larger cell killing probability than the same dose deposited by photons. This increased relative biological effectiveness is due

\footnotetext{
* Contribution to the Topical Issue "Atomic Cluster Collisions (7th International Symposium)", edited by Gerardo Delgado Barrio, Andrey V. Solov'yov, Pablo Villarreal, Rita Prosmiti.

a e-mail: p.devera@qub.ac.uk

b On leave from A. F. Ioffe Physical Technical Institute, 194021 St. Petersburg, Russian Federation.
}

to the large energy deposited around ion tracks on the nanoscale, giving place to an increase in the clustering of damaging events in biomolecules, especially in nuclear DNA, which makes the repair processes less effective [4].

The fundamental aspects of the problem are also of great interest. The irradiation with ions involves new physical phenomena which are not always considered properly (or considered at all) in biophysical models. In fact, the physical and chemical mechanisms underlying IBCT are complex, involving many different space, energy, and time scales, ranging from the transport of energetic ions in macroscopic tissues, the production of secondary electrons and radicals that can propagate on both the nanoand microscale (molecular and cellular levels, respectively) and their interaction with biomolecules on the nanometer scale leading to the final biological outcomes, noticeable in larger space and time scales [3]. More significantly all the physico-chemical processes occurring at the molecular level make it necessary to deviate from a simple energy deposition scheme. On these spatial scales not only energy deposition events are of importance, since the way in which this energy promotes different processes can significantly affect the final effects. While many processes are fairly well-known, such as the electron production and propagation or the generation of free radicals, new interactions are being discovered, such as the dissociative electron attachment, a mechanism by which very low energy electrons (with energies even below the ionization threshold) can fragment biomolecules [5]. 
In this context another damage mechanism has been theoretically predicted: ion-induced shock waves on the nanometer scale [6-8]. Ion beams can deposit large amounts of energy per unit path length (a carbon ion in the Bragg peak region deposits $900 \mathrm{eV} / \mathrm{nm}$ ) and the major part of this energy is used to eject secondary electrons of very low energies, below $50 \mathrm{eV}[3,9,10]$. Most of these secondary electrons transfer their energy to electronic excitations of the medium in less than a nanometer and the time scale over which this energy loss occurs is very short, of a few femtoseconds [11]. These times are very short in comparison with the mechanism capable of dissipating this energy, the electron-phonon coupling, which occurs on the sub-picosecond scale [11]. This situation results in a large heating of the medium in nanocylinders around the ion tracks, providing the conditions for a violent explosion of these "hot cylinders", a mechanism we refer to as ion-induced shock waves.

This shock wave effect was first predicted in terms of a hydrodynamics model where it was shown that pressures up to tens of GPa can be produced around ion tracks [7]. However, even if these high pressures suggest a possibility of biomolecular damage, this information is not sufficient for a detailed quantification, and subsequently molecular dynamics simulations were used to show how these conditions are sufficient to produce bond breaking in nucleosomes [6]. Moreover since the shock waves travel at high velocity they can propagate secondary species (i.e., free radicals, solvated electrons) much faster than the diffusion mechanism. All these dynamical and thermomechanical effects can drastically change the physicochemical environment to which biomolecules are exposed during irradiation.

A proper understanding of the characteristics of shock waves requires more systematic studies, especially by the use of molecular dynamics, a technique that can assess their properties and consequences in more detail. Such studies will enhance the understanding of the biological role of ion-induced shock waves, as well as help in the design of possible experiments to verify their existence. In the present paper we report molecular dynamics simulations to study the main features of ion-induced shock waves and their effects on DNA by means of the MBN Explorer software [12]. We focus the work on shock waves produced around carbon (one of the most promising projectiles used in IBCT) and iron ion tracks in the Bragg peak region. We study the dependence of several quantities, namely pressure waves and energy deposition in DNA bonds, on the size and characteristics of the system, in order to characterize the simulation box for future more systematic studies. These results are compared with an analytical hydrodynamics model [7] as well as with previous simulation results [6] to benchmark the present simulations. As a probe for biodamage we will use short DNA segments. This is because such short DNA duplexes can be simulated more straightforwardly than nucleosomes, so they are more convenient for systematic studies. Also, since the effects of shock waves are more noticeable over short distances, a short DNA strand should be enough for evaluating biomolecular damage, in a similar fashion to the effects of secondary electrons on DNA studied previously [3].

The methodology of the work is explained in Section 2, where the hydrodynamics model (Sect. 2.1) and the molecular dynamics procedure (Sect. 2.2) are discussed. The results of the simulations are presented in Section 3, where the pressure generated by the shock waves and their effects in the DNA duplex are shown. The final conclusions and remarks are given in Section 4.

\section{Methods}

Ion-induced nanoscopic shock waves were first described in terms of an analytical hydrodynamics model that was used for the first evaluation of their characteristics [7]. Even though it allows calculation of the basic physical features, such as the pressure generated during the shock wave, it does not allow the evaluation of the characteristics related to biological effects, such as the damage of DNA molecules, for which an atomic level description is needed. This kind of analysis can be performed through molecular dynamics simulations [6]. However, the analytical hydrodynamics model serves as a good benchmark for the molecular dynamics simulations. Some relevant features of the analytical hydrodynamics model are reviewed in Section 2.1. The molecular dynamics simulation technique is reviewed in Section 2.2. The choice of the biomolecular probe, as well as its setting up for the simulations, is explained in Section 2.2.1, and the setting up of the initial conditions for the simulation of the ion-induced shock wave is described in Section 2.2.2.

\subsection{Hydrodynamics model}

This model was adapted from a classical hydrodynamics treatment of the self-similar flow of liquid water and heat transfer [13] and applied to the specific situation of the energy transferred around an energetic ion track on the nanoscale where the resulting water flow is cylindrical [7].

Several predictions of this model are very convenient for benchmarking the simulations performed in the present work. In particular the radial position of the wave front $R$ as a function of time $t$ is given by:

$$
R(t)=\beta \sqrt{t}\left[\frac{S}{\rho}\right]^{1 / 4},
$$

and the pressure of the front $P_{\text {front }}$ as a function of the front radius $R$ is:

$$
P_{\text {front }}(R)=\frac{\beta^{4}}{2(\gamma+1)} \frac{S}{R^{2}} .
$$

In both equations, $\rho=1 \mathrm{~g} / \mathrm{cm}^{3}$ is the density of unperturbed liquid water, $\gamma=C_{\mathrm{p}} / C_{\mathrm{v}}=1.222$ for liquid water, $S$ is the stopping power (i.e., the mean energy loss per unit path length) of an ion in liquid water and $\beta$ is a parameter whose value for liquid water and the specific cylindrical geometry of the problem is $\beta=0.86$ [7]. 


\subsection{Molecular dynamics simulation}

In the molecular dynamics technique [14] all the atoms of the system are considered and their classical trajectories are followed by computing the interaction forces between them. The evolution with time $t$ of the coordinates $\boldsymbol{r}_{\boldsymbol{i}}(t)$ of each atom $i$ of mass $m_{i}$ is numerically computed for discrete time steps $\mathrm{d} t$, according to the Langevin equation:

$$
m_{i} \frac{\mathrm{d}^{2} \boldsymbol{r}_{i}}{\mathrm{~d} t^{2}}=\sum_{j \neq i} \boldsymbol{F}_{i j}-\frac{1}{\tau_{\mathrm{d}}} m_{i} \boldsymbol{v}_{i}+\boldsymbol{f}_{\boldsymbol{i}},
$$

where $\sum_{j \neq i} \boldsymbol{F}_{i j}$ is the total force acting on atom $i$ as a consequence of its interaction with all other atoms $j$ in the system (i.e., Newton's second law). The second and third terms on the right hand side of equation (3) correspond to the thermostat, used to keep the temperature of the system nearly constant to $T$, when coupled to a thermal bath. In the present work the Langevin thermostat is used which exerts a viscous force on each particle of velocity $\boldsymbol{v}_{\boldsymbol{i}}$, as well as a random force $\boldsymbol{f}_{\boldsymbol{i}}$ which guarantees thermal equilibrium. $\tau_{\mathrm{d}}$ is the damping time of the thermostat, while $\boldsymbol{f}_{\boldsymbol{i}}$ is a Gaussian random force with zero mean and variance $\sigma_{i}^{2}=2 m_{i} k_{\mathrm{B}} T / \tau_{\mathrm{d}}$ with $k_{\mathrm{B}}$ being the Boltzmann's constant.

For biomolecular systems, where the structure of the molecule is determined not only by interatomic distances but also by the geometric configuration of groups of atoms due to the molecular orbital hybridization, it is common to use special forcefields describing such interactions. In the CHARMM forcefield [15], one of the most common for describing biomolecules, the force acting on the atom $i$ is obtained from the potential energy $U(\boldsymbol{R})$ as $\sum_{j \neq i} \boldsymbol{F}_{i j}=\mathrm{d} U(\boldsymbol{R}) / \mathrm{d} \boldsymbol{r}_{\boldsymbol{i}}$ which corresponds to a given set of atomic coordinates $\boldsymbol{R}$ and is expressed as a combination of energies arising from the distances between pairs of bonded atoms, the angles formed between groups of three sequentially bonded atoms, the dihedral torsion angle formed by groups of sequentially four bonded atoms, the improper angles formed between groups of atoms that should form a plane, and the nonbonded interactions represented by the pure Coulomb force and the van der Waals interaction between pairs of atoms:

$$
\begin{aligned}
U(\boldsymbol{R})= & \sum_{\text {bonds }} K_{\mathrm{b}}\left(b-b_{0}\right)^{2}+\sum_{\text {angles }} K_{\theta}\left(\theta-\theta_{0}\right)^{2} \\
& +\sum_{\text {dihedr. }} K_{\chi}(1+\cos n \chi-\delta)+\sum_{\text {improp. }} K_{\varphi}\left(\varphi-\varphi_{0}\right)^{2} \\
& +\sum_{i} \sum_{j \neq i} \frac{q_{i} q_{j}}{\varepsilon r_{i j}}+\left[\epsilon_{i j}\left(\frac{R_{\min , i j}}{r_{i j}}\right)^{12}-\left(\frac{R_{\min , i j}}{r_{i j}}\right)^{6}\right] .
\end{aligned}
$$

In this equation $b$ is the bond distance between two bonded atoms, $\theta$ is the bond angle between every triplet of sequentially bonded atoms, $\chi$ is the dihedral torsion angle formed by every four atoms connected via covalent bonds and $\varphi$ is the improper torsion angle, used to maintain planarity between groups of sequentially bonded atoms; $b_{0}, \theta_{0}$ and $\varphi_{0}$
Table 1. Summary of the different simulation boxes used for the shock wave simulations. The four systems have a length of $4.6 \mathrm{~nm}$ along the track direction $y$, where PBC are applied.

\begin{tabular}{cccc}
\hline System & Shape & $\begin{array}{c}\text { Path-to-boundary } \\
\text { Distance }(\mathrm{nm})\end{array}$ & $\begin{array}{c}\text { PBC } \\
(x, z)\end{array}$ \\
\hline I & Disc & 10 & No \\
II & Parallelepiped & 10 & Yes \\
III & Disc & 17 & No \\
IV & Parallelepiped & 17 & Yes \\
\hline
\end{tabular}

correspond to the equilibrium quantities, while $n$ and $\delta$ determine the periodicity of the dihedral interaction. $K_{b}$, $K_{\theta}, K_{\chi}$ and $K_{\varphi}$ are the corresponding force constants. The Coulomb interaction is characterized by the atomic partial charges $q_{i}$, the interatomic distances $r_{i j}$ and the effective dielectric constant $\varepsilon$. The van der Waals interaction is defined by a 6-12 Lennard-Jones potential with well depth $\epsilon$ and the minimum energy distance $R_{\min }$. All of these parameters can be obtained for many biological molecules, including nucleic acids and proteins, from the CHARMM potential [15]. All simulations in this work have been performed using the CHARMM implementation within the MBN Explorer simulation package [12].

\subsubsection{Setting up the biological system}

Liquid water is the main constituent of living tissues. Therefore when we refer to the ion-induced shock waves in a cellular system they occur mainly in water. However, if we want to estimate possible biological effects, we should consider as a target of the shock wave some biological molecules, for example DNA. Thus in order to set up a simulation box for the present study we need to define (i) the water box in which the shock wave is going to propagate and (ii) the biological molecule that we are going to use to assess biodamage.

Point (i), even though it seems straightforward, has to be considered carefully. This is because the shock wave is a violent dynamical process and the pressure waves generated can propagate quickly, reaching, under certain circumstances, the boundaries of the simulation box. Such "boundary effects" can produce artifacts in the simulations. These artifacts could be avoided simply by setting an extremely large water box in order to guarantee that the shock wave will not arrive at the boundaries. However, the computational cost of molecular dynamics simulations increases with the number of atoms in the simulation box, so the size of the system has to be selected properly in order to perform the simulations in reasonable times.

In particular we want to study the effect of the size of the system in the simulation results as well as the inclusion of periodic boundary conditions to simulate extended media. For this reason in the present study we have constructed four different simulation boxes. These four systems are summarized in Table 1. The first box, which we will refer as system I, was designed as a liquid water disc of $10 \mathrm{~nm}$ radius around the ion's path, located in the disc center. This system is quite convenient since 


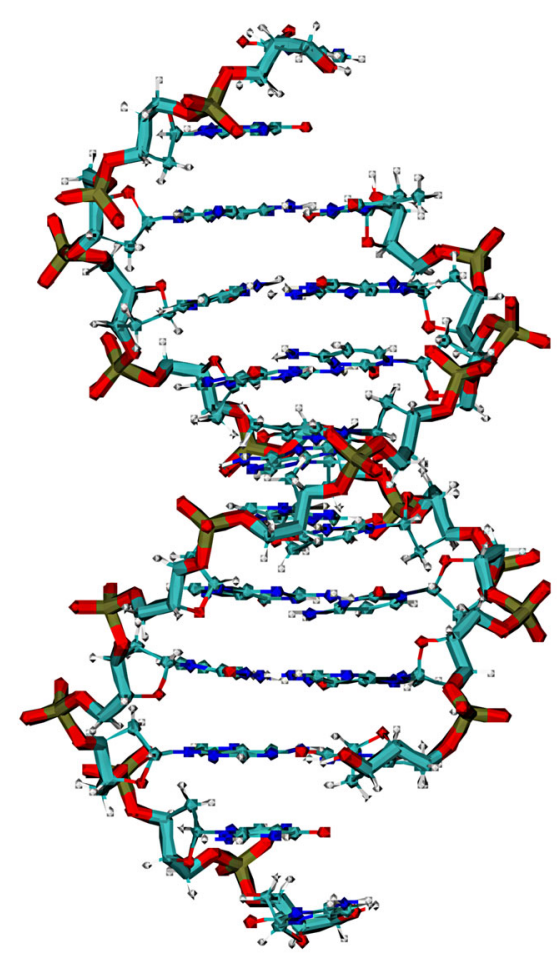

Fig. 1. Representation of the B-DNA molecule from the Protein Data Bank [16] with PDB ID 309D [17]. Thick lines represent the backbone bonds whose energy is monitored during the shock wave simulations (see Sect. 3).

it is quite small and thus computationally efficient. A radius of $10 \mathrm{~nm}$ was chosen following results from the hydrodynamics model, which show that the pressure of the shock wave is already very small after $10 \mathrm{~nm}[7]$. This disc, $4.6 \mathrm{~nm}$ long in the track direction $y$, was simulated with periodic boundary conditions (PBC) only in this direction. In order to check the effect of the inclusion of PBC in the directions $x$ and $z$, the parallelepiped system II was built. It has the same length of $4.6 \mathrm{~nm}$ in the track direction $y$ and the distance from the ion's path to the boundary is also $10 \mathrm{~nm}$ but $\mathrm{PBC}$ are applied in the three coordinates. Finally, to check size effects, two similar systems but with a path-to-boundary distance of $17 \mathrm{~nm}$ where built, systems III and IV. System IV will be used as a benchmark for the rest of them since it is the largest one and it has $\mathrm{PBC}$ in all directions.

Regarding point (ii), in the present work we would like to chose a simple system that can be simulated straightforwardly for performing more systematic studies of shock wave effects in biomolecules. As a first approximation we will use short DNA segments, as was done in earlier studies evaluating the damaging effect of secondary electrons [3]. They are simpler to treat than nucleosomes and, since shock wave effects are quite local, they should be large enough for estimating DNA damage.

In the present study we have chosen the B-DNA molecule from the Protein Data Bank [16] with PDB ID 309D [17]. This molecule, represented in Figure 1, consists on a B-DNA duplex containing eight base-pairs and two free nucleotides at each 5'-end. These sticky ends are complementary, forming continuous 10 -fold double helix molecules. This molecule is very convenient since it allows the building of DNA duplex models as long as desired, by the replication and displacement of the original molecule as many times as wanted. It opens, as well, the possibility of being used as a building block for more complex DNA structures if desired.

Currently we have used the 309D molecule to construct a linear DNA duplex, each strand being 30-base long, which we consider is enough for an initial assessment of the shock wave effects in DNA. After building this molecule from the original one from the Protein Data Bank it is solvated in liquid water and an atmosphere of 60 sodium counterions is placed around the DNA (since each base carries one negative charge) by means of the software VMD [18]. Then, using MBN Explorer [12], the system is optimized by a velocity quenching algorithm, and equilibrated at $T=310 \mathrm{~K}$ (body temperature), using the Langevin thermostat with damping time $\tau_{\mathrm{d}}=0.2 \mathrm{ps}$, a simulation time step $\mathrm{d} t=1 \mathrm{fs}$, periodic boundary conditions, the particle mesh Ewald algorithm for the longrange Coulomb interactions, and a van der Waals cut off distance of $13 \AA$. The geometry of the resulting DNA duplex, as well as the structure of the sodium ions environment, has been checked and compared with reference data [19-21]. The equilibrated DNA duplex has then been put in the different water boxes described above for the subsequent shock wave simulations. The DNA duplex, oriented in the $z$ direction, is always placed with its center at $2 \mathrm{~nm}$ distance from the ion's path, in such a way that the closest DNA atom is $\sim 1 \mathrm{~nm}$ from the path. The initial configurations for systems I and II are shown in Figures 2a and $2 \mathrm{~d}$.

\subsubsection{Setting up the initial conditions of the shock wave}

An energetic ion losses its energy mainly by electronic excitations and ionizations. From the large number of ionization events a large number of low energy electrons are produced (below $50 \mathrm{eV}$ in the Bragg peak) $[3,9,10,22]$ which will propagate on the nanometer scale. The dynamics of such a process was studied in reference [11], where it was shown how the radial dose around the ion track is built up in 50 fs. Almost all the energy lost by the ion is deposited within $1 \mathrm{~nm}$ from the ion path. It is well known that electron-phonon coupling, the mechanism by which the energy deposited by the secondary electrons can be dissipated, occurs in times much longer than fs, i.e., in the sub-ps scale. This means that a large amount of energy will be locally deposited within $\sim 1 \mathrm{~nm}$ very quickly ( $\sim 50 \mathrm{fs})$ and that it will be released at once, setting the initial conditions for the formation of the shock wave.

In the molecular dynamics simulations we select the water molecules initially present within $1 \mathrm{~nm}$ radius from the ion path. These molecules are highlighted in Figure 2, where their atoms are shown as spheres. All the energy lost 

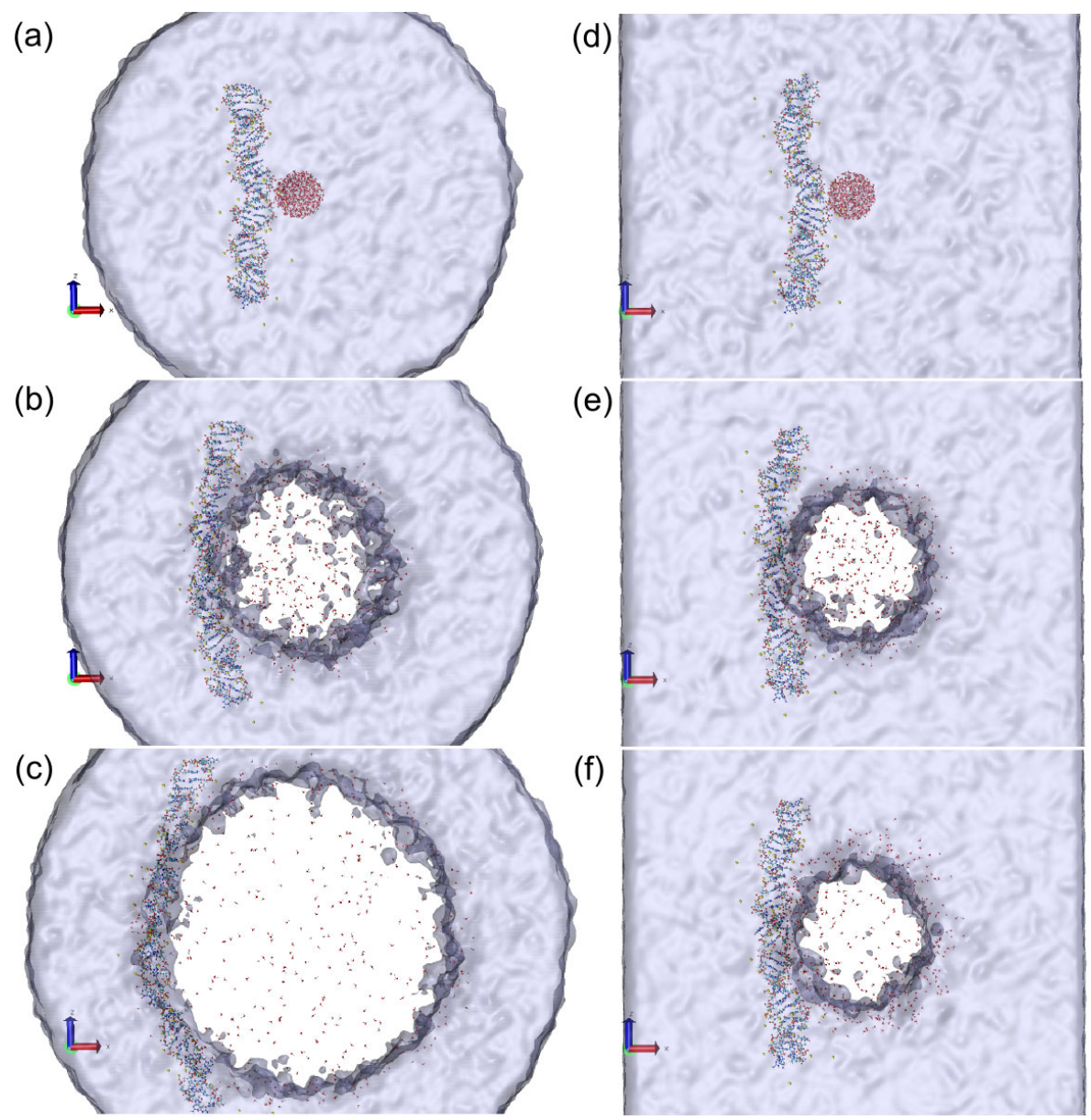

Fig. 2. Snapshots of a shock wave induced by a carbon ion in the Bragg peak region in liquid water with the ion's path ( $y$ direction) crossing systems I and II at $1 \mathrm{~nm}$ distance from the DNA duplex (oriented in the $z$ direction). Panels (a), (b), and (c) correspond to the system I at 0,5 , and $10 \mathrm{ps}$ after the ion traversal, while panels (d), (e), and (f) represent the same times for the system II. The water molecules initially excited by the ion track as well as the DNA duplex are highlighted, with their atoms shown as explicit atoms.

by the ion (which is not considered explicitly in the simulations since it crosses the system in much shorter times) will be transferred to these molecules so the velocities of their atoms $v_{i}$ (obtained from previous equilibration) are multiplied by a factor $\alpha$, in a way in which their total kinetic energy after the ion crosses the system is:

$$
\sum_{i}^{N} \frac{1}{2} m_{i}\left(\alpha \cdot v_{i}\right)^{2}=\frac{3 N k_{\mathrm{B}} T}{2}+S l
$$

The first term on the right hand side of the equation corresponds to the initial kinetic energy of the excited cylinder (with $N$ atoms) at an equilibrium temperature $(T=310 \mathrm{~K})$. The second term is the energy lost by the ion when crossing the system, which is its stopping power $S$ times the length of the simulation box $l$. The simulation of the shock wave is performed as indicated previously for the equilibration but without a thermostat and with lower values of the time step $\mathrm{d} t$, depending on the simulation, to ensure conservation of energy.

\section{Results and discussion}

We will start our study of the effects of ion-induced shock waves in DNA duplexes with a carbon ion in the Bragg peak region (with kinetic energy of $\sim 300 \mathrm{keV} / \mathrm{u}$ ), carbon being a common choice in modern ion beam therapy centers. The stopping power of a carbon ion in the Bragg peak is $900 \mathrm{eV} / \mathrm{nm}$. Figures 2a-2c show three snapshots of the evolution of the system after irradiation $(0,5$, and $10 \mathrm{ps}$, respectively) when using system I, i.e., a $10 \mathrm{~nm}$ radius disc in vacuum (see Tab. 1 ). The same results are shown in Figures 2d-2f for system II, i.e., a parallelepiped of $10 \mathrm{~nm}$ path-to-boundary distance with periodic boundary conditions (PBC).

The explosion of the system is much more violent for system I than for system II with the structure of the DNA duplex being heavily distorted in Figure 2c. This is due to the high pressures produced by the shock wave that force the system to expand into vacuum in system I. However, this behavior does not occur in system II, apparently because the periodic boundary conditions damp the effects 
of the shock wave. The final structure of the DNA duplex is distorted in system II (Fig. 2f) but not as much as in the case of system I. Therefore, periodic boundary conditions are needed to suppress the system explosion if a small system is used for the simulations.

A better understanding of this situation can be achieved by calculating the pressure generated by the shock wave. These values can be compared to the results provided by the analytical model (Sect. 2.1). To calculate the pressure, virtual walls have been placed at different radial distances $r$ from the ion's path. At several times $t$ during the simulation the number of atoms crossing this wall in each direction has been monitored and their momentum $p_{i}=m_{i} v_{i}$ calculated. The pressure is calculated as:

$$
P=\frac{\mathrm{d} p}{\mathrm{~d} t A}=\frac{2\left(\sum_{i} p_{i}-\sum_{j} p_{j}\right)}{\mathrm{d} t A}
$$

where $\mathrm{d} t$ is the time passed between frames in the simulation, $A$ is the surface of the cylindrical wall and the indexes $i$ and $j$ refer to the atoms crossing the wall in the outer and in the inner directions respectively. The factor 2 comes from the assumption that, if the wall was there to measure the pressure, the atoms would have an elastic collision coming back after the collision with the same momentum $p_{i}$ but in nearly opposite direction so the momentum transfer would be $\mathrm{d} p_{i} \simeq 2 p_{i}$.

The results for the time evolution of the pressure wave produced by a carbon ion in the Bragg peak in system IV (where boundary effects are not expected) are shown in Figure 3a. The wave front can be clearly identified during the first picosecond, where the pressure profile has a sharp maximum that propagates rapidly (faster than $\sim 1600 \mathrm{~m} / \mathrm{s}$ ) in the radial direction. After the first few picoseconds the pressure wave widens and loses intensity quickly. This time evolution can be compared with the results of the hydrodynamics model (Sect. 2.1). This comparison is shown for the position of the front and its pressure, respectively, in Figures $4 \mathrm{a}$ and $4 \mathrm{~b}$, where the lines are the results from the analytical model and symbols represent the results from the simulations (which have been obtained by finding the maximum of the Gaussian fitting each curve in Fig. 3a). It should be noted that, in this work, we define the wave front as the point where the pressure profile presents a maximum. This definition is slightly different from the one corresponding to the hydrodynamics model, since within this model the front is always a sharp maximum followed by a wake, while the medium beyond the front is unperturbed (see Ref. [7]). However, molecular dynamics simulations account for the widening of the front as time passes and this is the reason why we define it here as the position of the maximum pressure. In any case, the good comparison of the simulations and the analytical model, as it will be seen bellow, support this definition.

The first important observation is that simulation results in system IV follow the results of the analytical model for carbon. However, artifacts appear in the results of systems II and III due to their small size or the lack of PBC. In the case of system II, where the path-to-boundary dis-

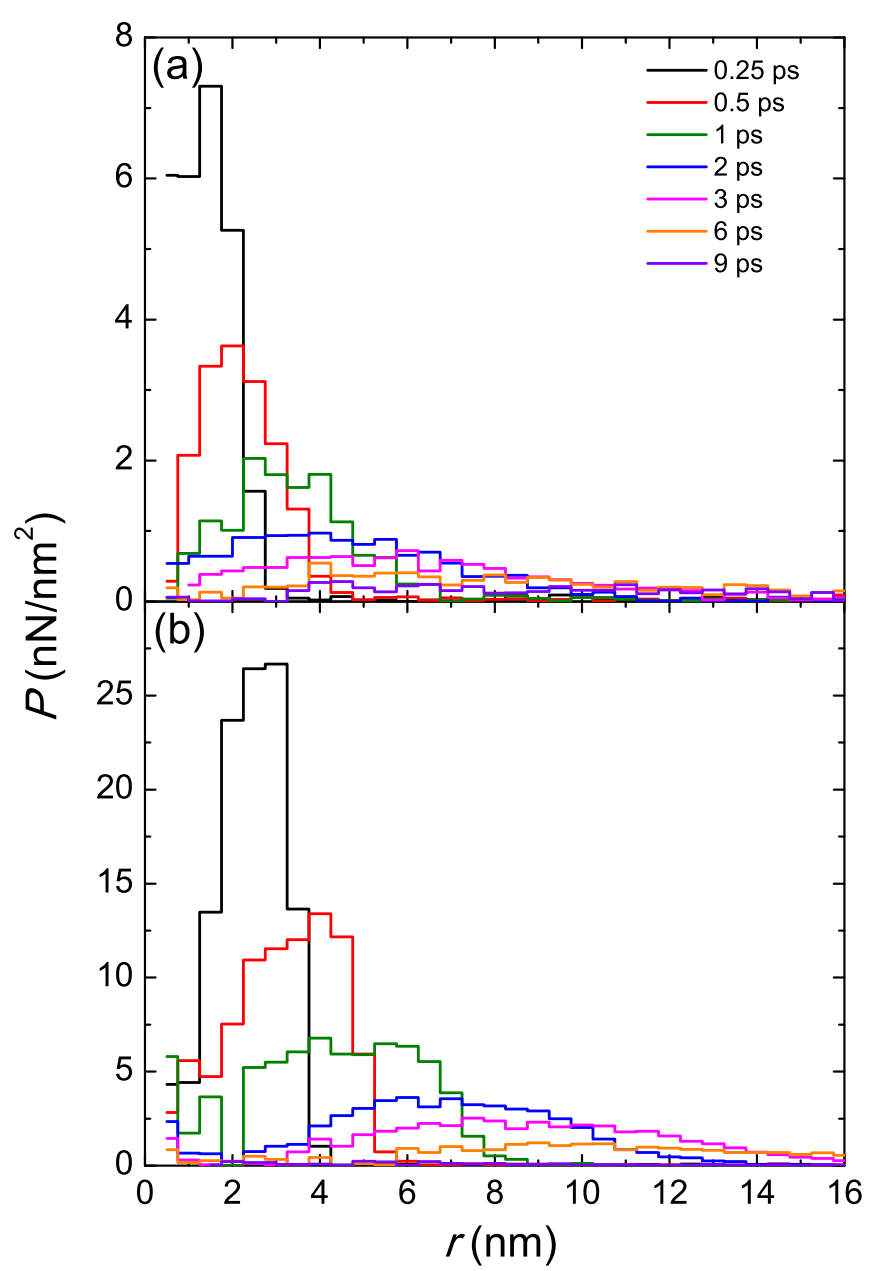

Fig. 3. Time evolution of the pressure, as a function of the radius from the path $r$, generated by (a) a carbon ion and (b) an iron ion in the Bragg peak region in system IV, a parallelepiped with $17 \mathrm{~nm}$ path-to-boundary distance and periodic boundary conditions (see Tab. 1).

tance is just $10 \mathrm{~nm}$, the tail of the pressure wave reaches the boundary after $\sim 3$ ps. This produces an interference of the pressure wave in the boundary, which stops the front propagation. Even though the disc-like system III has a path-to-boundary distance of $17 \mathrm{~nm}$, the lack of $\mathrm{PBC}$ seems to produce an unnatural propagation of the front at longer times. Neither of these effects are observed for carbon when system IV is used, which suggests that the system has to be large enough and PBC should be applied in order to properly contain the shock wave.

Before discussing results for a heavier ion (iron), we will analyze the effects of the carbon-induced shock wave in the DNA duplex. We have monitored the energy stored in the covalent bonds of the DNA backbone closest to the track [6]. The inset in Figure 5a shows the potential energy of some of the covalent bonds (first term on the right hand side of Eq. (4)), initially located within $2.2 \mathrm{~nm}$ of the ion's path, as a function of time. The energy stored in the bonds varies in time, with some sudden jumps due to the exposure of the bonds to the pressure of the shock 


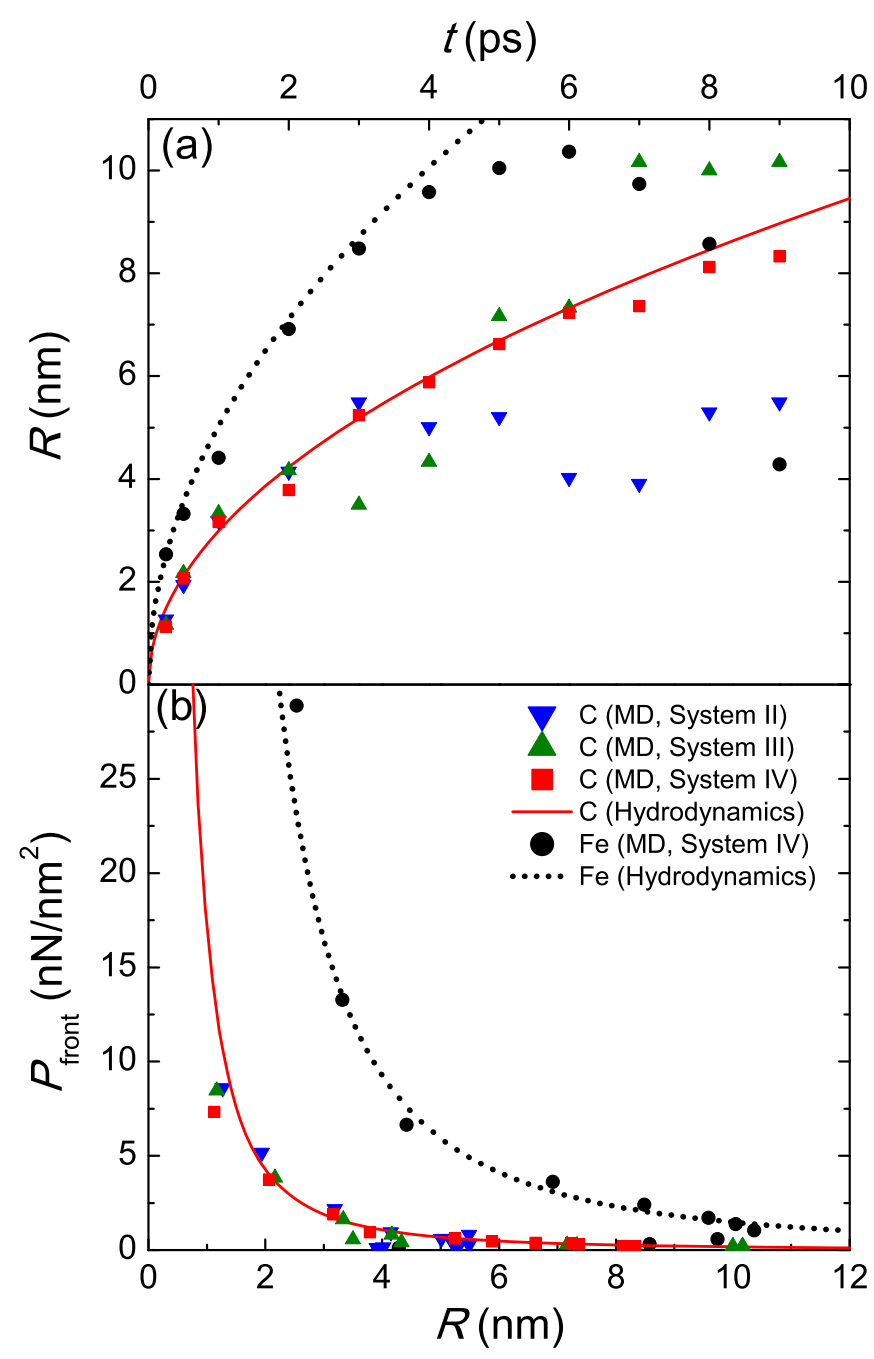

Fig. 4. (a) Time evolution of the wave front position for carbon and iron ion induced shock waves in the Bragg peak region. (b) Pressure of the wave front as a function of its radial position, for carbon and iron induced shock waves in the Bragg peak region. Symbols represent molecular dynamics results, while lines are the predictions of the analytical hydrodynamics model. See the text for further details.

wave. Each of those local maxima can be stored as an energy deposition event. The frequency count of such events for the shock wave produced by a carbon ion in the Bragg peak in all the systems I-IV is represented in Figure 5a. This frequency, which presents an exponential behavior, can be parameterized to estimate the probability for inducing single strand breaks, regarded as energy deposition events larger than some given threshold, typically around $2.5 \mathrm{eV}$ [6]. Clearly system I presents a very different behavior while systems II-IV converge. This result arises from the artificial violent explosion of system I, due to the lack of pressure to damp the shock wave. The convergence between systems II-IV emphasizes the importance of the first picoseconds of the shock wave in terms of DNA damage: even though the pressure wave differs in these systems after $\sim 3 \mathrm{ps}$, their energy deposition profile

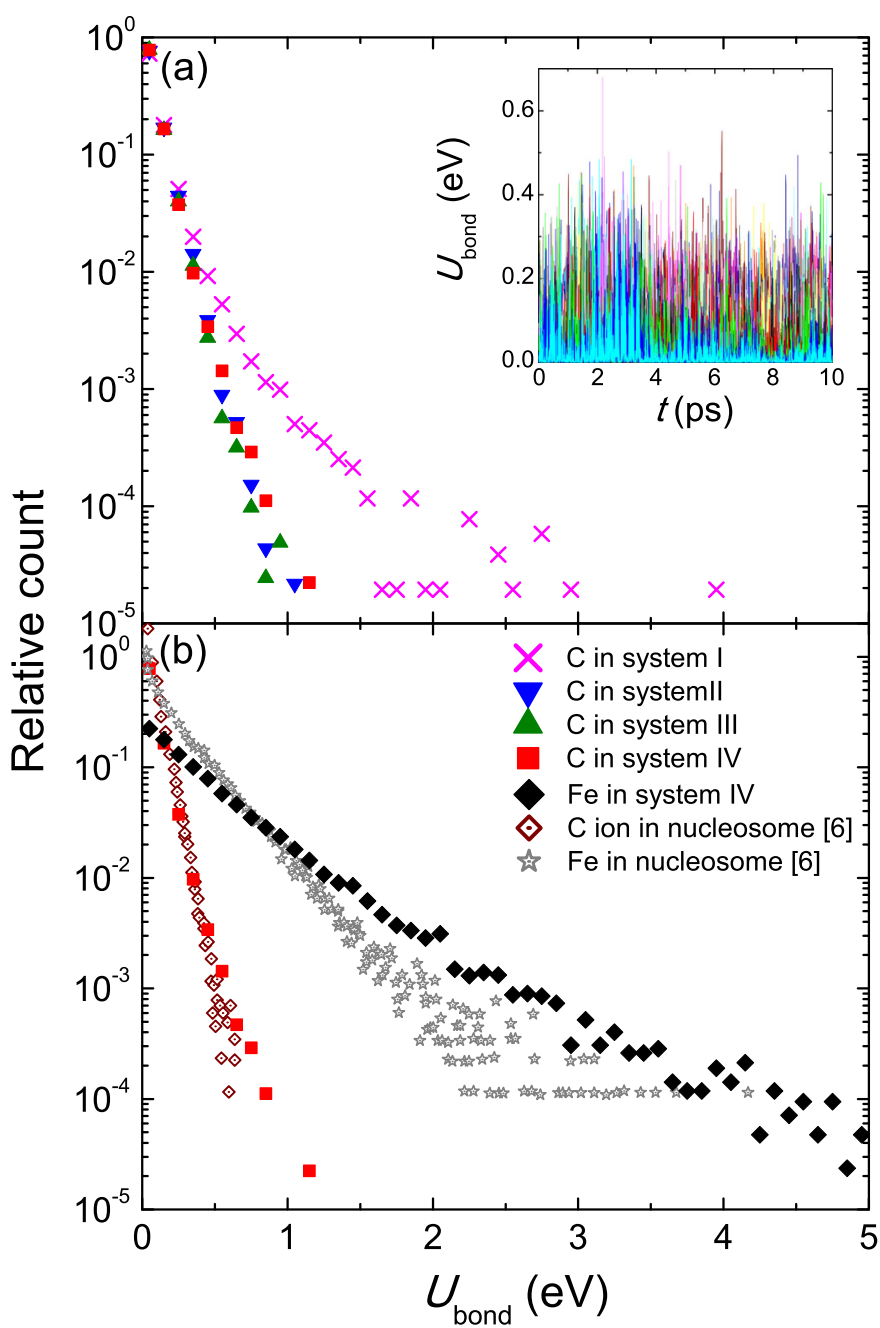

Fig. 5. Relative count of energy deposition events in the DNA backbone bonds located within $2.2 \mathrm{~nm}$ of the ion's path. (a) Present results for a carbon ion in the Bragg peak region in systems I-IV. (b) Present results for carbon and iron ions in system IV, compared with previous results in the nucleosome [6]. The inset shows the time evolution of the potential energy of some of the bonds analyzed for a carbon ion in system IV.

is similar, highlighting the fact that it is built up during the first picoseconds after the explosion. The energy deposition profile is compared with previous simulations in the nucleosome [6] in Figure 5b, where we find a fairly good match, allowing for the different geometries.

From these simulations it is clear that there is a relatively low probability of producing single strand breaks by the shock wave in the case of carbon ions (assuming a SSB threshold of $2.5 \mathrm{eV}$; however, such thresholds may be even lower [6]). In terms of a possible experimental verification of these results it would be convenient to study ions with larger stopping powers since these can produce larger numbers of strand breaks, so they are easier to detect. For this reason we have performed a simulation of the shock wave produced by an iron ion in the Bragg peak region, having a stopping power of $7195 \mathrm{eV} / \mathrm{nm}$. System IV 

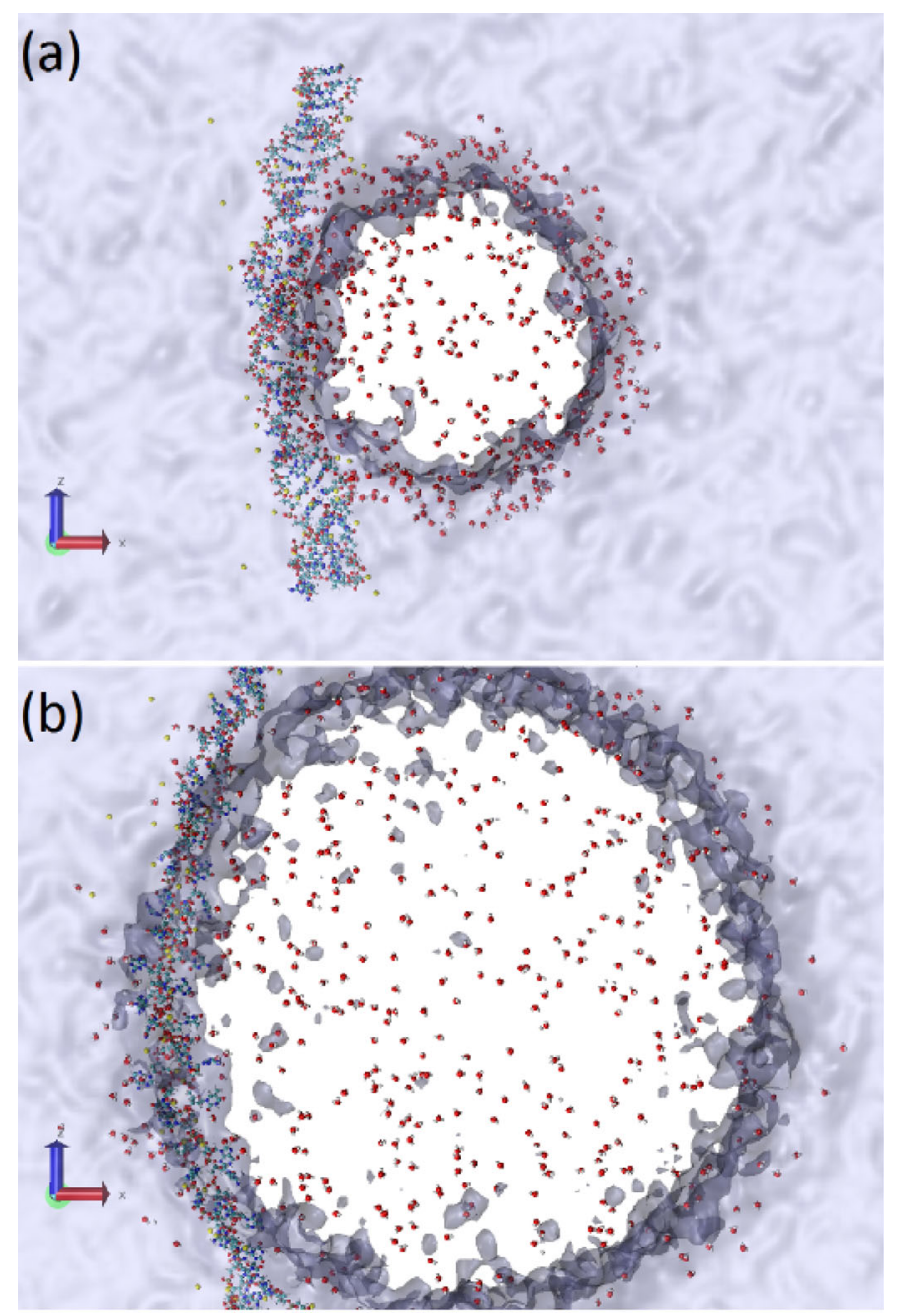

Fig. 6. Geometry of system IV, 10 ps after passage of (a) a carbon ion and (b) an iron ion, in the Bragg peak region. Nanochannels are formed in liquid water, and the DNA structure is distorted, particularly heavily in the case of the iron ion.

has been used for this simulation since, attending to the results for carbon, the other systems would be too small. The evolution of the pressure wave for iron is shown in Figure $3 \mathrm{~b}$, the evolution of the front is depicted in Figure 4, and the energy deposition profile in the DNA backbone bonds is shown in Figure 5b. The final geometry of the system after $10 \mathrm{ps}$, as compared with the carbon case, is shown in Figure 6.

Both the position of the front and its pressure, shown in Figure 4, seem to follow the predictions of the analytical model but only up to $4 \mathrm{ps}$, after which the front actually goes backwards. This behavior is caused by the interference of the pressure wave at the boundaries of the system, as was observed for carbon with system II. In order to check the influence of the size of the system in the simulation results, we performed two more simulations of the shock wave produced by the iron ion in two different systems, one with a path-to-boundary distance of $15 \mathrm{~nm}$, and another one of $20 \mathrm{~nm}$. From these simulations (these results are not shown in the figures for clarity), the time at which the front goes backwards shortens as the size of the system decreases, confirming that this effect is an artifact of the simulations due to the limited size of the simulation box. As expected from equations (1) and (2), the velocity of propagation of the iron shock wave is 1.682 times faster than for carbon and the pressure of the front is 8 times larger. This results from the fact that the stopping power for iron in the Bragg peak region is 8 times larger than for carbon.

Such large pressures lead to larger distortions of the DNA duplex being observed for iron in comparison with carbon in Figure 6. This leads to a larger number of high energy deposition events in the DNA backbone bonds, as shown in Figure 5b, where the slope of the curve is much smaller than for carbon ion and many more events larger than $2.5 \mathrm{eV}$ (a conservative estimation for the threshold for single strand break production) are produced. This would justify the use of heavier ions for a possible experimental verification of shock wave effects, where more single strand breaks can be detected. The results for iron are compared with previous simulations performed for the nucleosome [6]. In this case, the present results are not that close to the previously reported values. However, the order of magnitude seems to be similar. The observed differences could be due to several reasons, the most probable being the different geometry. The larger slope of the previously reported data suggests that the histone protein has some protective role in DNA damage. In the present work the DNA duplex is free, allowing a larger stretching of the molecule which might be impeded in the nucleosome. Also the fact that system IV is not large enough for the iron-induced shock wave could be important. However, as seen from the results for carbon, the first picoseconds of the simulation are the most relevant for the DNA damage and the molecular dynamics simulation follows properly the analytical results up to $4 \mathrm{ps}$. It is clear, in any case, that a larger system is needed for the simulation of an iron ion shock wave. This has not been done here in order not to increase the computational expense of the calculations. However, precautions should be taken in future calculations to optimize the box for each ion. In any case, the main conclusion that can be extracted from Figure 4 is that molecular dynamics simulations can reproduce perfectly the results predicted by the analytical hydrodynamics model as long as the system used for the simulations is large enough for containing the wave and PBC are applied. Indeed the hydrodynamics model can be used as a consistency check to analyze whether the system built for molecular dynamics simulation is appropriate or not.

\section{Conclusions}

In this paper we have presented a molecular dynamics study of the effects of ion-induced shock waves in biological media. The study focuses on the pressure waves arising from the heating of liquid water after energetic carbon and iron ions traversal (in the Bragg peak region) and on the effects of such waves on short DNA duplexes. 
The first important conclusion of this paper is that molecular dynamics simulations of ion-induced shock waves can reproduce the results obtained by the analytical hydrodynamics model [7], in terms of front velocity and pressure, as long as the simulation box is properly designed. This point is of great importance: ion-induced shock waves have not been detected yet experimentally and their existence has been so far only predicted theoretically. The fact that independent techniques, such as classical molecular dynamics and classical hydrodynamics, agree in the prediction of the properties of the shock waves adds arguments in favor of their existence and establishes the theoretical grounds over which possible experiments can be designed.

Molecular dynamics simulations reproduce the analytical results only if the system is well designed. This means that it has to be large enough to contain the pressure waves produced and that preferably periodic boundary conditions should be applied to avoid boundary effects in the simulation, such as the interference of the pressure wave in the periodic boundaries or the expansion of the system into vacuum when $\mathrm{PBC}$ are not applied. In the present case, a path-to-boundary distance of $17 \mathrm{~nm}$ has been demonstrated to be large enough for carbon-induced shock waves in the Bragg peak region. For iron larger boxes should be used in future work. However, smaller boxes can be used for certain simulations as long as one is interested in short times and distances from the ion's path where most of the damage occurs. The analytical hydrodynamics model can be always used to check the consistency of the results, as shown in this paper.

The energy deposition profile in the DNA backbone bonds has been analyzed, both for carbon and iron ions, as an estimation of possible biological damage. The results obtained for carbon are very similar to those reported previously for molecular dynamics simulations in the nucleosome [6]. In the case of iron ions we have found some differences with the previous published data. However, the order of magnitude of the results is quite similar, confirming the systematics of the stopping power of the ion on the expected damage of DNA. The observed differences are most likely due to the different geometry of the systems used, where the presence of the histone protein in the nucleosome can have some protective effect.

The present results establish a solid procedure for performing more systematic simulations of shock wave effects in DNA duplexes, where the analytical hydrodynamics model can be used as a benchmark. The properties of the shock waves for ions of different stopping powers can be predicted and their effects on DNA determined. This opens the door to new simulation improvements, such as the inclusion of reactive force fields for a better prediction of DNA damage $[23,24]$ or the study of the shock wave effects in the propagation of secondary species generated around ion tracks. Such systematic studies will allow a better understanding of the biological relevance of ion-induced shock waves and will be useful for the future design of potential experiments for their detection.

\section{Author contribution statement}

The molecular dynamics simulations were performed by $\mathrm{PdV}$ under the supervision of AVS. The manuscript was mainly drafted by $\mathrm{PdV}$, following discussions with AVS, NJM and FJC. All authors contributed to the conceptual design of the work, actively participated in the discussion of the results, provided valuable comments and insight, and contributed to the revision of the manuscript.

The authors acknowledge financial support from the European Union's FP7-People Program (Marie Curie Actions) within the Initial Training Network No. 608163 "ARGENT", Advanced Radiotherapy, Generated by Exploiting Nanoprocesses and Technologies. PdV also acknowledges additional support from the European Regional Development Fund and the Spanish Ministerio de Economía y Competitividad (project No. FIS2014-58849-P). The molecular dynamics simulations were performed at the computer clusters IMACT, at The Open University (UK), and KELVIN, at Queen's University Belfast (UK).

\section{References}

1. J.S. Loeffler, M. Durante, Nature Rev. Clin. Oncol. 10, 411 (2013)

2. M. Scholz, T. Elsässer, Adv. Space Res. 40, 1381 (2007)

3. E. Surdutovich, A.V. Solov'yov, Eur. Phys. J. D 68, 353 (2014)

4. D. Schardt, T. Elsässer, D. Schulz-Ertner, Rev. Mod. Phys. 82, 383 (2010)

5. B. Boudaïffa, P. Cloutier, D. Hunting, M.A. Huels, L. Sanche, Science 287, 1658 (2000)

6. E. Surdutovich, A.V. Yakubovich, A.V. Solov'yov, Scientific Reports 3, 1289 (2013)

7. E. Surdutovich, A.V. Solov'yov, Phys. Rev. E 82, 051915 (2010)

8. A.V. Yakubovich, E. Surdutovich, A.V. Solov'yov, Nucl. Instrum. Methods B 297, 135 (2012)

9. P. de Vera, R. Garcia-Molina, I. Abril, A.V. Solov'yov, Phys. Rev. Lett. 110, 148104 (2013)

10. P. de Vera, I. Abril, R. Garcia-Molina, A.V. Solov'yov, J. Phys.: Conf. Ser. 438, 012015 (2013)

11. E. Surdutovich, A.V. Solov'yov, Eur. Phys. J. D 69, 193 (2015)

12. I.A. Solov'yov, A.V. Yakubovich, P.V. Nikolaev, I. Volkovets, A.V. Solov'yov, J. Comp. Chem. 33, 2412 (2012)

13. L. Landau, E. Lifshitz, Fluid Mechanics, 2nd edn. (ReedElsevier, Oxford, Boston, Johannesburg, 1987), Vol. 6 of Course of Theoretical Physics

14. M.P. Allen, D.J. Tildesley, Computer simulation of liquids (Oxford University Press, Oxford, 1989)

15. A.D. MacKerell Jr. et al., J. Phys. Chem. B 102, 3586 (1998)

16. H.M. Berman, J. Westbrook, Z. Feng, G. Gilliland, T.N. Bhat, H. Weissig, I.N. Shindyalov, P.E. Bourne, Nucl. Acids Res. 28, 235 (2000)

17. H. Qiu, J.C. Dewan, N.C. Seeman, J. Mol. Biol. 267, 881 (1997) 
18. W. Humphrey, A. Dalke, K. Schulten, J. Mol. Graph. 14, $33(1996)$

19. U. Heinemann, C. Alings, M. Bansal, EMBO J. 11, 1931 (1992)

20. F. Pan, C. Roland, C. Sagui, Nucl. Acids Res. 42, 13981 (2004)

21. T.J. Robbins, Y. Wang, J. Biomolec. Struct. Dynam. 31, 1311 (2013)

22. O.I. Obolensky, E. Surdutovich, I. Pshenichnov, I. Mishustin, A.V. Solov'yov, W. Greiner, Nucl. Instrum. Methods B 266, 1623 (2008)
23. D. Bottländer, C. Mücksch, H.M. Urbassek, Nucl. Instrum. Methods B 365, 622 (2015)

24. G.B. Sushko, I.A. Solov'yov, A.V. Verkhovtsev, S.N. Volkov, A.V. Solov'yov, Eur. Phys. J. D 70, 12 (2016)

Open Access This is an open access article distributed under the terms of the Creative Commons Attribution License (http://creativecommons.org/licenses/by/4.0), which permits unrestricted use, distribution, and reproduction in any medium, provided the original work is properly cited. 